\title{
Direct detection of concealed mineral deposits through microbial community fingerprinting
}

B.P. IUlianella Phillips ${ }^{1 *}$, R.L. SimisteR ${ }^{2}$, P.A. WINTERBURN $^{\dagger}$, S.A. CROWE ${ }^{2}$

${ }^{1}$ MDRU, University of British Columbia, Vancouver, V6T

1Z4, Canada (*correspondance: bphillips@eoas.ubc.ca)

${ }^{2}$ Microbiology and Immunology, University of British

Columbia, Vancouver, V6T 1Z3, Canada

'Deceased

Mineral exploration in northern latitudes is challenging in that undiscovered deposits are likely buried beneath appreciable glacial overburden. The development of innovative exploration strategies and robust techniques to see through cover is imperative to future mineral deposit discovery success.

Microbial communities are sensitive to subtle environmental gradients, and they will reflect these gradients through spatial variability. Variations in microbial community profiles, induced by chemical and physical differences related to geology, are detectable and can be used to vector toward discrete geological features. The modernization of DNA sequencing and big-data evaluation allows for efficient and cost-effective microbial fingerprinting of soil profiles, with the potential to see through glacial cover to find buried ore mineralization.

Results to date demonstrate viability of microbial fingerprinting to directly identify the sub-crop of mineralization in addition to entrained geochemical signatures in till. Microbial community profiling was conducted on soils above two copper-porphyries and an epithermal deposit in British Columbia, two kimberlites in the Northwest Territories, and a variety of soil-incubation experiments. These analyses reveal significant microbial community shifts, correlated with the presence of subsurface mineralization, with a distinct species-level community response over ore mineralization. The relationship between microbial community profiles and mineralization supports the application of microbial fingerprinting as a method to accurately detect subsurface geological features, such as the presence of ore deposits in glacially covered terrain.

The integration of microbial community information with soil chemistry and landscape development coupled with geology and geophysics can improve the drill / no-drill decision process. There is thus strong potential for application of microbial fingerprinting in the mineral deposit exploration industry. 